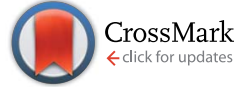

Cite this: RSC Adv., 2016, 6, 55715

Received 11th March 2016 Accepted 24th May 2016

DOI: $10.1039 / \mathrm{c} 6 \mathrm{ra06510d}$

www.rsc.org/advances

\section{Radical exchange reaction of multi-spin isoindoline nitroxides followed by EPR spectroscopy $\dagger$}

\author{
I. Wessely, ${ }^{a}$ V. Mugnaini, ț A. Bihlmeier, ${ }^{c}$ G. Jeschke, ${ }^{d}$ S. Bräse ${ }^{\text {ae }}$ and M. Tsotsalas ${ }^{\star a b}$ \\ The synthesis of a rigid, isoindoline-functionalized tetraphenylmethane multi-spin system is described. The \\ isoindoline nitroxide groups are used in a nitroxide exchange reaction with a TEMPO containing \\ alkoxyamine. Using EPR spectroscopy it is possible to follow the exchange process and thereby find the \\ optimal experimental conditions to have the maximum yield. The presented approach could be used to \\ study the nitroxide exchange process of various systems and to determine the kinetics of the exchange \\ process. The presented molecular components can be used as tectons in the construction of covalently \\ linked organic networks or as model systems for EPR distance measurements.
}

\section{Introduction}

The synthesis of rigid organic building blocks containing multiple functional groups is of high interest to create molecular tectons for the synthesis of crystalline or amorphous covalently linked materials as well as for the assembly of non-covalently bound supramolecular architectures. ${ }^{1}$ The functionalization of these molecular tectons with nitroxide moieties lead to attractive model systems for EPR distance measurements ${ }^{2}$ and would allow the assembly of the tectons via halogen bonding ${ }^{3}$ or nitroxide exchange reaction. ${ }^{4}$ The nitroxide exchange reaction, ${ }^{5}$ which belongs to the class of dynamic covalent chemistry, has been incorporated in dynamic polymers or macromolecules ${ }^{6}$ and as a tool to trigger the self-assembly of micro-crystals. ${ }^{7}$ In addition, the nitroxide exchange reaction has been utilized to functionalize polymers, self-assembled monolayers ${ }^{8}$ or surfaces. ${ }^{9}$ Moreover, the incorporation of profluorescent nitroxides allows following the kinetics of the nitroxide exchange reaction. ${ }^{4}$ However, in all the reported cases, only the product formation could be followed, not the exchange process as such. Herein we report the synthesis of a rigid tetrahedral organic tecton

${ }^{a}$ Institute of Organic Chemistry (IOC), Karlsruhe Institute of Technology (KIT), Fritz-Haber-Weg 6, D-76131 Karlsruhe, Germany.E-mail: manuel.tsotsalas@kit.edu ${ }^{b}$ Institute of Functional Interfaces (IFG), Karlsruhe Institute of Technology (KIT), Hermann-von-Helmholtz-Platz 1, D-76344 Eggenstein-Leopoldshafen, Germany 'Institute of Physical Chemistry (IPC), Karlsruhe Institute of Technology (KIT), FritzHaber-Weg 2, D-76131 Karlsruhe, Germany

${ }^{d}$ ETH Zurich, Laboratory of Physical Chemistry, Vladimir-Prelog-Weg 2, CH-8093 Zurich, Switzerland

${ }^{e}$ Institute of Toxicology and Genetics (ITG), Karlsruhe Institute of Technology (KIT), Hermann-von-Helmholtz-Platz 1, D-76344 Eggenstein-Leopoldshafen, Germany

$\uparrow$ Electronic supplementary information (ESI) available: Materials and methods, synthetic procedures, details on EPR measurements/fits. See DOI: $10.1039 / \mathrm{c} 6 \mathrm{ra06510d}$

\$ Current address: International Iberian Nanotechnology Laboratory, Avenida Mestre José Veiga, 4715-330, Braga, Portugal. containing multiple nitroxide moieties. Afterwards we performed a nitroxide exchange reaction and demonstrate that progress of the reaction can be followed by EPR spectroscopy.

\section{Results and discussion}

The synthesis of the rigid multi-spin molecule is based on tetrakis(4-azidophenyl)methane (1) as a core, which is functionalized with nitroxide moieties via fourfold copper-catalysed azide alkyne click chemistry ${ }^{10}$ between the azide functions of the core $\mathbf{1}$ and the alkyne moiety of an isoindoline nitroxide $\mathbf{2}$. Scheme 1 shows the synthesis of tetraphenylmethane nitroxide (TPM-NO) 3. The TPM-NO 3 can be further functionalized using dynamic and reversible nitroxide exchange reaction. Nitroxide exchange reactions are based on thermal $\mathrm{C}-\mathrm{O}$ bond homolysis of alkoxyamines, which leads to transient carbon-centred radicals and persistent nitroxide radicals. Usually these carbon centred radicals are quickly trapped by the nitroxide radicals and reform the alkoxyamines. If homolysis of an alkoxyamine is performed in presence of an additional nitroxide radical, also this additional nitroxide can trap the carbon centred nitroxide, and a mixture of the two possible nitroxides will be formed. The ratio will depend on the relative thermodynamic stabilities of the different alkoxyamines (see Fig. 1 for a schematic representation of the bond homolysis and of the nitroxide exchange reaction). ${ }^{11}$

If the EPR (electron paramagnetic resonance) spectra of the two nitroxide moieties (depicted as red and green in Fig. 1) differ, i.e. in the hyperfine coupling constant as in the present case, the exchange process can be followed via EPR measurements. In order to evaluate the possibility to follow the exchange process between the isoindoline nitroxide of TPM-NO 3 and 2,2,6,6-tetramethylpiperidin-1-yloxyl (TEMPO, 5), we recorded the EPR spectra of the individual compounds and mixtures thereof in toluene at room temperature. 


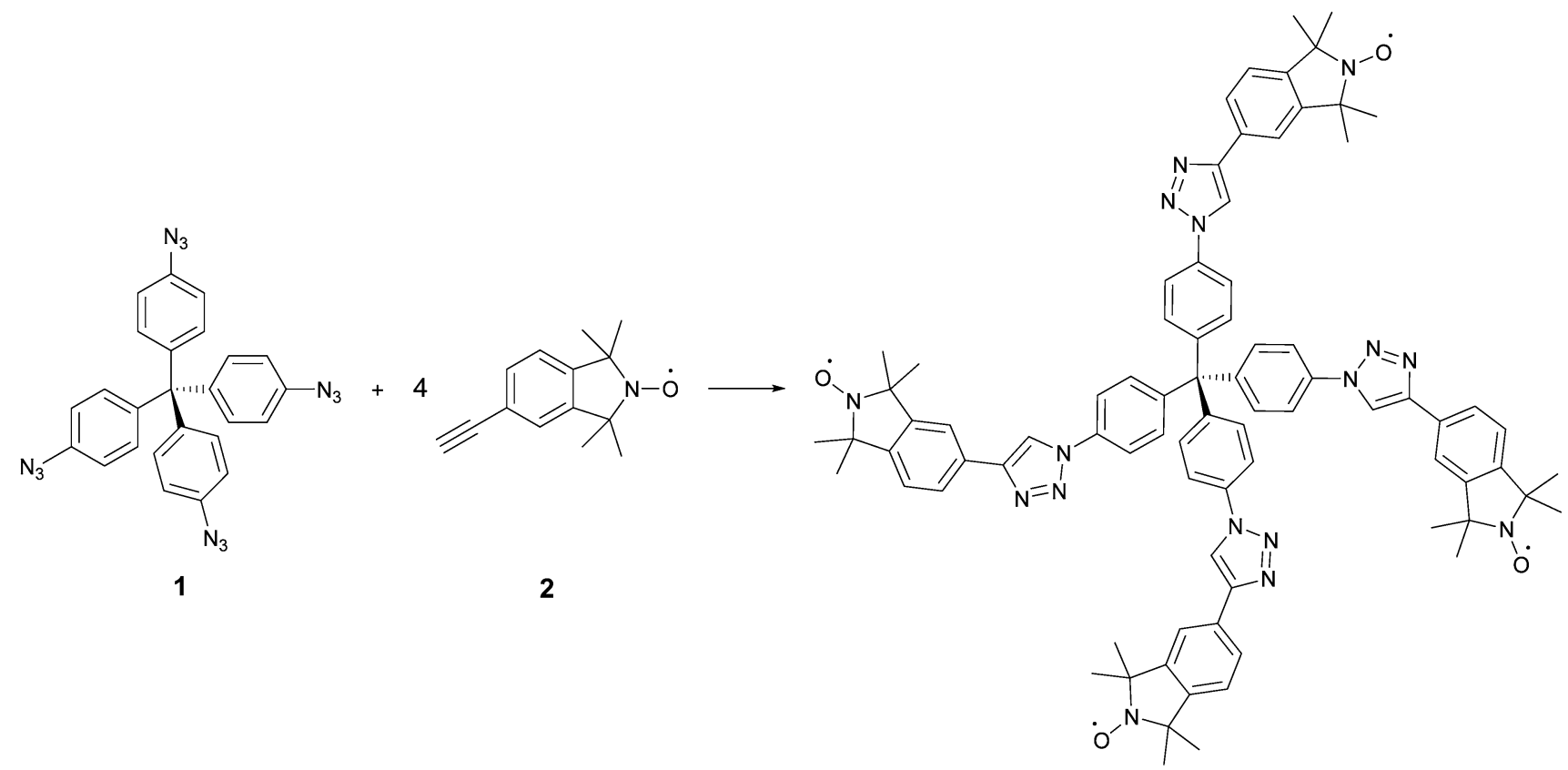

3

Scheme 1 Synthesis of TPM-NO 3 using fourfold copper-catalysed azide alkyne click chemistry.

a)

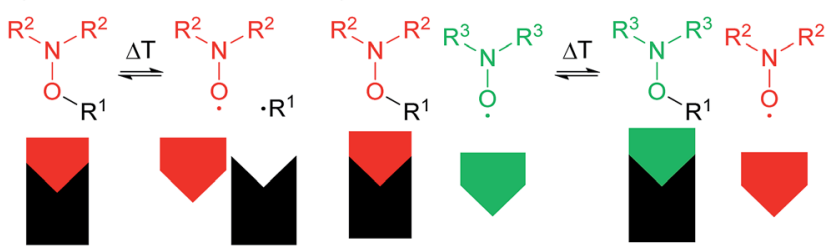

Fig. 1 (a) Homolysis of an alkoxyamine into a nitroxide radical (red) and a carbon centred radical (black); (b) thermodynamic product formation for homolysis of an alkoxyamine in presence of an additional nitroxide radical (green).

Fig. 2 shows the continuous wave (CW) EPR spectra of TPMNO 3 (left) and TEMPO 5 (right) and mixtures of TPM-NO 3/ TEMPO 5 with molar ratios of $9 / 1,1 / 1$ and 1/9. As can be seen from the EPR spectra in Fig. 2, the isoindoline nitroxide moiety of TPM-NO 3 has a hyperfine coupling constant of about 14.1 Gauss.
The EPR spectra show the typical tumbling induced line profile. The TEMPO nitroxide 5 instead has a hyperfine coupling constant of about 15.5 Gauss. Due to the difference in the hyperfine coupling constants of the isoindoline and TEMPO nitroxides, the exchange reaction can be easily followed by means of EPR.

We performed the nitroxide exchange reaction of the multispin system TPM-NO 3 with the TEMPO-alkoxyamine 4 shown in Scheme 2. Firstly, we followed the exchange reaction between TPM-NO 3 and TEMPO-alkoxyamine 4 using an equimolar ratio (with respect to the nitroxide moieties). Compounds 3 and 4 were dissolved in toluene and mixed at room temperature. The resulting solution was divided in several aliquots, inserted in closed ampules and degassed via bubbling with argon. Afterwards the different aliquots were heated under argon at $80{ }^{\circ} \mathrm{C}$ for either $15 \mathrm{~min}, 1 \mathrm{~h}, 2 \mathrm{~h}, 14 \mathrm{~h}, 48 \mathrm{~h}$ or $96 \mathrm{~h}$. After cooling to room temperature the continuous wave EPR spectra were recorded. Fig. 3 (top) shows the CW EPR spectra for the different points in time.

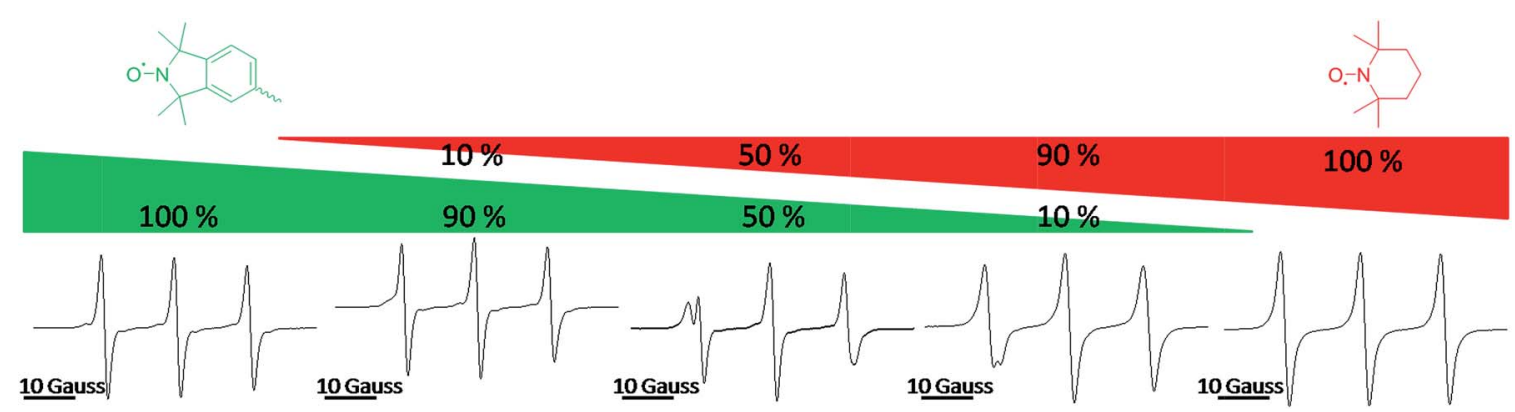

Fig. 2 Continuous wave (CW) EPR spectra of TPM-NO 3 (left) and TEMPO 5 (right) and mixtures of TPM-NO 3/TEMPO 5 with molar ratios 9/1, 1/1 and $1 / 9$. 


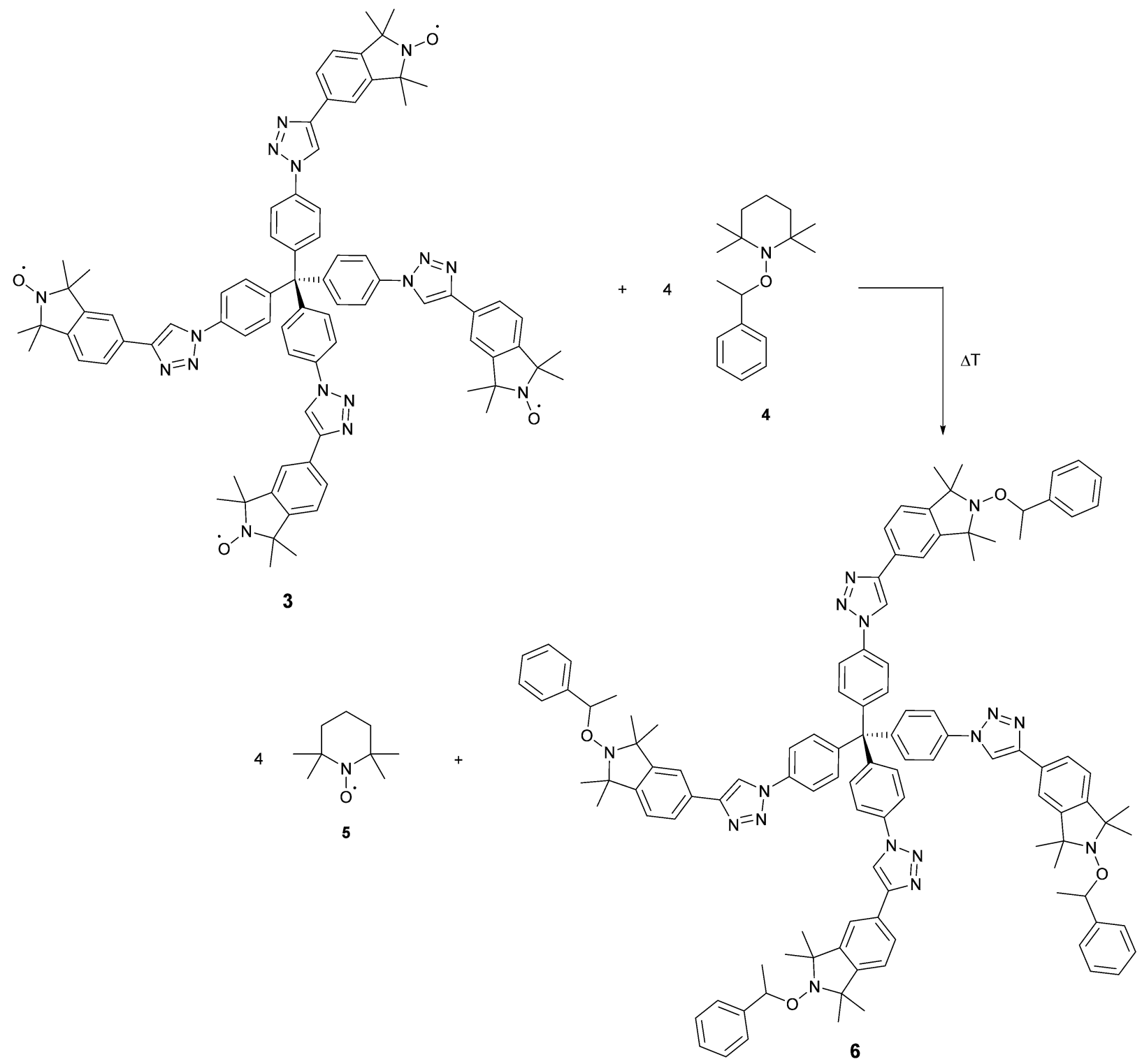

Scheme 2 Nitroxide exchange reactions of TPM-NO 3 and TEMPO-alkoxyamine 4.

In the EPR spectra of Fig. 3 (top), one can clearly see the progress of the exchange reaction over time, with a decrease in relative intensity of the species with lower hyperfine coupling constant (TPM-NO 3) and an increase of TEMPO 5, showing a larger hyperfine coupling constant. From the fitting of the spectra (for details see the ESI $\dagger$ ), we could obtain the different relative percentages of the two species and could plot the exchange percentage as function of time (see Fig. 3, bottom). The plot reveals that the exchange process is a fast initial reaction (within the first two hours) followed by a deceleration. The deceleration is most likely due to the decreasing concentration of TEMPO-alkoxyamine 4 in combination with the competition for radical trapping between an increasing concentration of liberated TEMPO 5 and decreasing concentration of TPM-NO 3.

Even after $96 \mathrm{~h}$, the exchange reaction does not seem to have reached the thermodynamic limit, where both species adapt their final equilibrium concentrations. In order to find conditions under which the equilibrium is reached faster and in order to optimize the yield of TPM-NO-alkoxyamine 6, we investigated the influence of both the reaction temperature and the equivalents of TEMPO-alkoxyamine 4 on the exchange process. Fig. 4 shows the comparison of the CW EPR spectra of TPM-NO 3 and TEMPO-alkoxyamine 4 in mixtures of $1: 1$ (a), $1: 2$ (b), and $1: 5$ (c) molar ratios (related to nitroxide moieties), after heating at $100{ }^{\circ} \mathrm{C}$ for $1 \mathrm{~h}$ and $24 \mathrm{~h}$.

The EPR spectra show that the exchange reaction is almost quantitative after $24 \mathrm{~h}$ at $100{ }^{\circ} \mathrm{C}$ in the presence of 5 equivalents of TEMPO-alkoxyamine 4 with $96 \%$ yield (and $77 \%$ yield after 1 $\mathrm{h}$ ), whereas the $1 / 1$ (20\% yield after $1 \mathrm{~h}$ and $65 \%$ after $24 \mathrm{~h}$ ) and $1 / 2$ mixtures (39\% yield after $1 \mathrm{~h}$ and $80 \%$ yield after $24 \mathrm{~h}$ ) still contain large amounts of isoindoline nitroxide moieties of TPM-NO 3. 

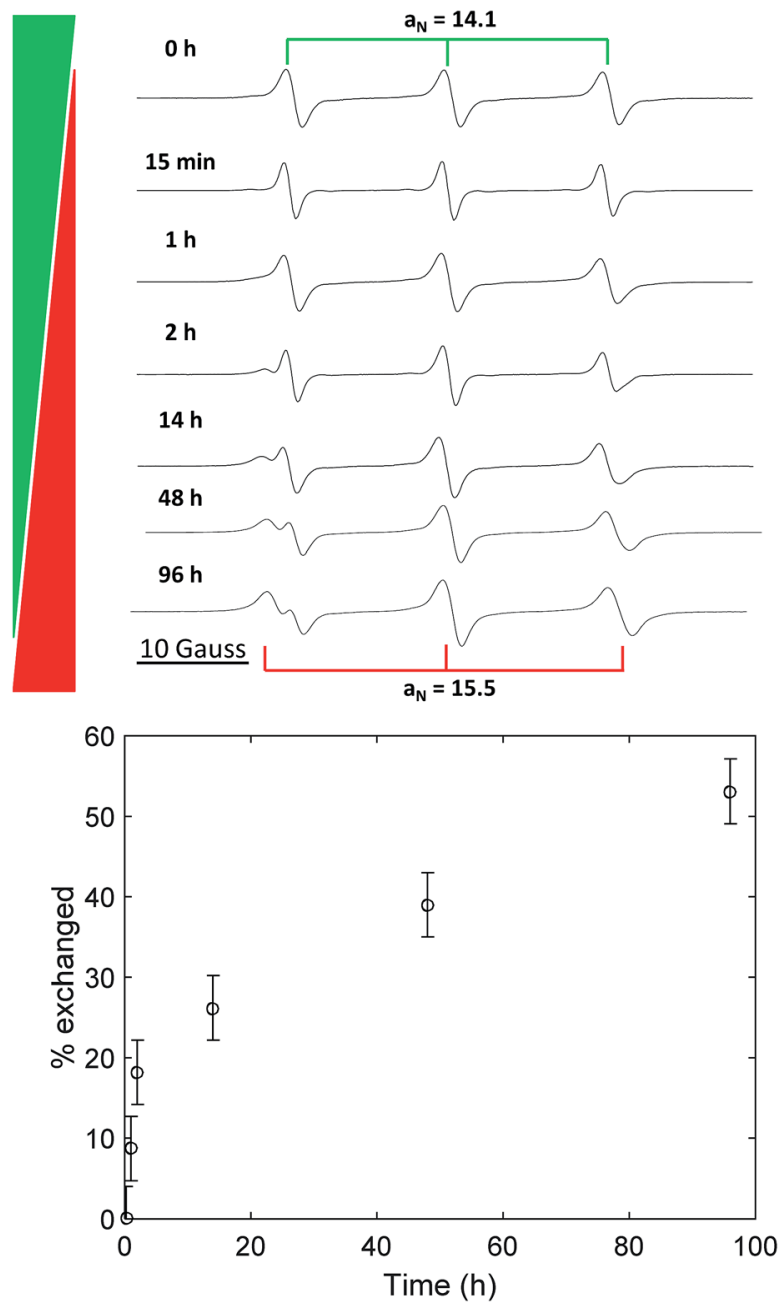

Fig. 3 Top: EPR spectra of TPM-NO 3/TEMPO-alkoxyamine 4 1/1 mixture in toluene without heating $\left(0^{\circ} \mathrm{C}\right)$ and after heating at $80^{\circ} \mathrm{C}$ for $15 \mathrm{~min}, 1 \mathrm{~h}, 2 \mathrm{~h}, 14 \mathrm{~h}, 48 \mathrm{~h}$ and $96 \mathrm{~h}$. Bottom: graph showing the percentage of exchanged nitroxides versus time.

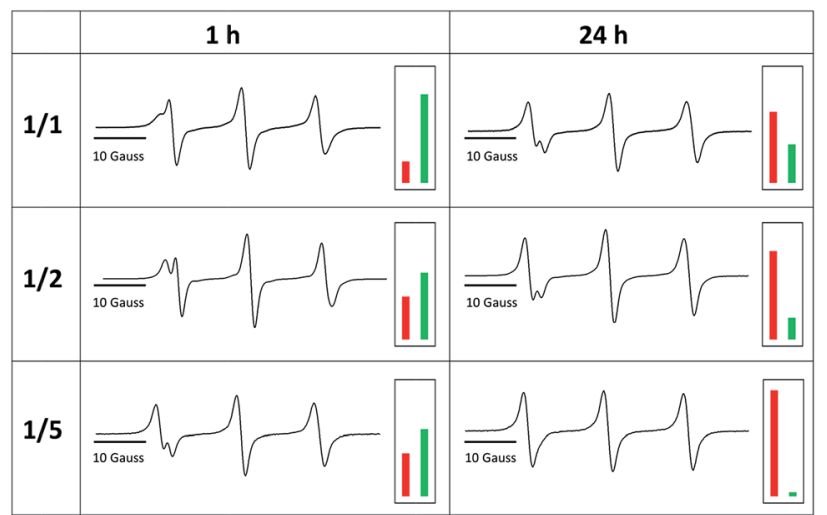

Fig. 4 CW EPR spectra of TPM-NO 3 and TEMPO-alkoxyamine 4 after heating at $100{ }^{\circ} \mathrm{C}$ for $1 \mathrm{~h}$ and $24 \mathrm{~h}$. Top: $1 / 1$ molar ratio of TPM-NO 3/ TEMPO-alkoxyamine 4; middle: $1 / 2$ molar ratio of TPM-NO 3/TEMPOalkoxyamine 4 ; bottom: $1 / 5$ molar ratio of TPM-NO 3/TEMPOalkoxyamine 4 .
Table 1 Calculated limit of exchange for the radical exchange process of TPM-NO 3 and TEMPO-alkoxyamine 4 compared with the experimental data after $24 \mathrm{~h}$ heating at $100^{\circ} \mathrm{C}$

\begin{tabular}{lllll}
$\begin{array}{l}\text { Molar } \\
\text { ratio } \\
\text { of 3/4 }\end{array}$ & $\begin{array}{l}\text { Calculated for } \\
\mathrm{kJ} \mathrm{mol}^{-1}\end{array}$ & $\begin{array}{l}\text { Calculated for } \\
\mathrm{kJ} \mathrm{mol}^{-1}\end{array}$ & $\begin{array}{l}\text { Calculated for } \\
\mathrm{kJ} \mathrm{mol}^{-1}\end{array}$ & $\begin{array}{l}\text { Experimental } \\
\text { after 24 h at } \\
T=100\end{array}$ \\
\hline $1 / 1$ & $66 \%$ & $58 \%$ & $50 \%$ & $65 \%$ \\
$1 / 2$ & $97 \%$ & $91 \%$ & $82 \%$ & $80 \%$ \\
$1 / 5$ & $100 \%$ & $100 \%$ & $100 \%$ & $96 \%$
\end{tabular}

To verify if the exchange reaction reaches equilibrium at $100{ }^{\circ} \mathrm{C}$ after $24 \mathrm{~h}$, we determined the expected thermodynamic limit of exchange for the investigated molar ratios. For this, we first estimated the Gibbs free energy $\Delta G$ by performing quantum chemical calculations. Employing density functional theory methods as implemented in the TURBOMOLE program package, ${ }^{12}$ we obtain values between $-4 \mathrm{~kJ} \mathrm{~mol}^{-1}$ and $+5 \mathrm{~kJ}$ $\mathrm{mol}^{-1}$. Considering the error in the calculated $\Delta G$, we evaluated the limit of exchange for several values in the range of $\pm 15 \mathrm{~kJ}$ $\mathrm{mol}^{-1}$ (for details of the calculations see Section 5 in the ESI $†$ ). The experimental results for $T=100{ }^{\circ} \mathrm{C}$ after $24 \mathrm{~h}$ and the calculated results for selected values of $\Delta G$ are summarized in Table 1 . The calculated results for slightly negative values of $\Delta G$ fit well to the experimentally observed data. The calculations suggest that the exchange process is approaching its thermodynamic limit of exchange after heating at $100{ }^{\circ} \mathrm{C}$ for $24 \mathrm{~h}$ and that a nearly quantitative exchange can be reached if the TEMPO-alkoxyamine $\mathbf{4}$ is present in excess.

\section{Experimental}

The EPR spectra in this work were recorded on a Bruker ESP300E spectrometer. The compounds were dissolved in toluene and deoxygenated by bubbling argon for several minutes. After treatment of the samples as written in the text the spectra were taken at $298 \mathrm{~K}$.

The instrument settings were as follows: microwave power $2.00 \mathrm{~mW}$, modulation amplitude $0.0452 \mathrm{mT}$, modulation frequency $100 \mathrm{kHz}$, scan time $180 \mathrm{~s}$. Further details as well as the synthesis of the compounds are described in the ESI. $\dagger$

\section{Conclusions}

Here we describe the successful synthesis of novel rigid isoindoline based multispin nitroxides via fourfold click reaction. These nitroxides were further converted into alkoxyamines via exchange reaction with a TEMPO-alkoxyamine and characterized using EPR spectroscopy. Our results demonstrate that EPR spectroscopy is a versatile tool to follow the exchange process and to determine several factors influencing the kinetics of the exchange process and optimize the experimental conditions to have the maximum yield. The presented approach could be used to study the nitroxide exchange process of various systems and the presented molecular components can be used as initiators for nitroxide mediated polymerization $(\mathrm{NMP})^{13}$ or as 
tectons in the construction of supramolecular and covalently linked organic networks. Further investigations in this regards are currently ongoing.

\section{Acknowledgements}

We acknowledge the SFB 1176 funded by the German Research Council (DFG) in the context of projects C5 (M. Tsotsalas) and Q4 (A. Bihlmeier) as well as the Studienstiftung and the CarlZeiss-Stiftung for funding. We thank Dr Laure Monnereau for authentic samples of $\mathbf{1}$ and Dr Fairful-Smith for authentic samples of 2 .

\section{Notes and references}

1 T. Muller and S. Bräse, RSC Adv., 2014, 4, 6886-6907.

2 S. Valera, J. E. Taylor, D. S. B. Daniels, D. M. Dawson, K. S. Athukorala Arachchige, S. E. Ashbrook, A. M. Z. Slawin and B. E. Bode, J. Org. Chem., 2014, 79, 8313-8323.

3 G. R. Hanson, P. Jensen, J. McMurtrie, L. Rintoul and A. S. Micallef, Chem.-Eur. J., 2009, 15, 4156-4164.
4 J. P. Blinco, K. E. Fairfull-Smith, A. S. Micallef and S. E. Bottle, Polym. Chem., 2010, 1, 1009-1012.

5 C. J. Hawker, G. G. Barclay and J. Dao, J. Am. Chem. Soc., 1996, 118, 11467-11471.

6 H. Otsuka, Polym. J., 2013, 45, 879-891.

7 B. Schulte, M. Tsotsalas, M. Becker, A. Studer and L. De Cola, Angew. Chem., Int. Ed., 2010, 49, 6881-6884.

8 H. Wagner, M. K. Brinks, M. Hirtz, A. Schäfer, L. Chi and A. Studer, Chem.-Eur. J., 2011, 17, 9107-9112.

9 M. Becker, L. D. Cola and A. Studer, Chem. Commun., 2011, 47, 3392-3394.

10 O. Plietzsch, C. I. Schilling, M. Tolev, M. Nieger, C. Richert, T. Muller and S. Brase, Org. Biomol. Chem., 2009, 7, 47344743.

11 H. Fischer, Chem. Rev., 2001, 101, 3581-3610.

$12 \mathrm{~V}$. TURBOMOLE, Program Package for ab initio Electronic Structure Calculations, a development of University of Karlsruhe and Forschungszentrum Karlsruhe GmbH 19892007, TURBOMOLE GmbH, 2007, http:// www.turbomole.com.

13 C. J. Hawker, A. W. Bosman and E. Harth, Chem. Rev., 2001, 101, 3661-3688. 